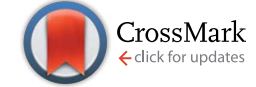

Cite this: RSC Adv., 2017, 7, 2211

Received 9th October 2016 Accepted 28th November 2016

DOI: $10.1039 / c 6 r a 24974 d$

www.rsc.org/advances

\section{Synthesis of graphene oxide/polyimide mixed matrix membranes for desalination $\uparrow$}

\begin{abstract}
Bo Feng, Kai Xu and Aisheng Huang*
Nanohybrid graphene oxide (GO)/polyimide (PI) mixed matrix membranes (MMMs) were prepared by dispersing GO into PI through wet phase inversion method. The structure of GO/PI MMMs was characterized by using XRD, FT-IR and XPS. FESEM was used to analyze the morphology of the GO/PI MMMs. The hydrophilicity of the GO/PI MMMs was determined by static water contact angles. The performances of the GO/PI MMMs were evaluated for desalination of seawater through pervaporation. The GO/PI MMMs display excellent water permeability and salt rejection for desalination of different concentrations of seawater. At $90{ }^{\circ} \mathrm{C}$, the GO/PI MMMs exhibit a high water flux $\left(36.1 \mathrm{~kg} \mathrm{~m}^{-2} \mathrm{~h}^{-1}\right)$ and a high salt rejection (99.9\%). Further, the GO/PI MMM displays a high stability for seawater desalination. This high separation performance combined with high stability and facile fabrication route recommend the developed GO/PI MMMs as a promising candidate for desalination of seawater.
\end{abstract}

\section{Introduction}

At present, the shortage of water resource has already become a worldwide problem because of the intense increase of water demand and severe aggravation of water pollution. Recently, considerable efforts have been afforded to obtain drinking water from abundantly available saline seawater. ${ }^{1}$ In this direction, the membrane-based separation technology like reverse osmosis (RO) has been recognized as a promising alternative for seawater desalination due to their lower energy consumption. ${ }^{2-4}$ However, the present membranes used for seawater desalination are mainly polymeric membranes, which usually suffer from biofouling, metal oxide fouling, abrasion and mineral scaling, thus resulting in low stability and low rejection. ${ }^{5,6}$ In comparison with organic polymer membranes, the inorganic membranes are more stable under harsh separation condition. So far, various inorganic membranes, such as carbon membrane, ${ }^{7,8}$ zeolite membranes, ${ }^{9-11}$ and metal organic frameworks membrane $^{\mathbf{1 2}}$ have been developed for seawater desalination. Despite much progress in the development of membranes for seawater desalination, it is still highly desired to develop a novel membrane with high stability and high ions rejection for seawater desalination. ${ }^{\mathbf{1 3 - 1 5}}$

Recently, two-dimensional graphene oxide (GO), with multifunctional surface chemistry, has drawn much attention for the fabrication of molecular sieve membranes. ${ }^{\mathbf{1 6}-18}$ Through simple

Institute of New Energy Technology, Ningbo Institute of Materials Technology and Engineering, Chinese Academy of Sciences, 1219 Zhongguan Road, 315201 Ningbo, P. R. China.E-mail: huangaisheng@nimte.ac.cn

$\dagger$ Electronic supplementary information (ESI) available: XPS, mechanical properties and desalination performances of the GO/PI MMMs. See DOI: 10.1039/c6ra24974d vacuum filtration of GO suspensions, molecular or ionic sieving GO membranes can be easily prepared for gas separation. ${ }^{\mathbf{1 9 - 2 2}}$ Further, as a derivative of graphene but containing oxygen-rich functional groups like hydroxyl, epoxy groups, the GO membranes show high hydrophilicity, ${ }^{23}$ leading to a high water permeability. ${ }^{17,18,24}$ Considering that the actual diffusion path of GO $(0.3 \mathrm{~nm})^{25}$ is just between the size of water molecules $(0.26$ $\mathrm{nm})$ and hydrated ions (e.g. $\mathrm{Na}^{+} 0.72 \mathrm{~nm}, \mathrm{~K}^{+} 0.66 \mathrm{~nm}, \mathrm{Ca}^{2+}$ $\left.0.82 \mathrm{~nm}, \mathrm{Mg}^{2+} 0.86 \mathrm{~nm}, \mathrm{Cl}^{-} 0.66 \mathrm{~nm}\right)$, GO membranes can show high salt rejections in seawater desalination. In fact, as reported previously, ${ }^{26,27}$ the GO membrane has displayed high desalination performances. However, since the mechanical strength of the GO membranes is not as good as polymeric membranes due to electrostatic repulsion ${ }^{28}$ or mechanical stress, ${ }^{29}$ the GO membrane is easily destroyed in the practical applications.

Attributing to the presence of abundant oxygen-rich functional groups, GO possesses strong interaction with the polymer chain, and thus is well dispersed into the polymer matrix. ${ }^{30-32}$ Therefore, the mechanical strength and stability of GO film can be enhanced when graphene oxide is modified by crosslinkers ${ }^{26,27,33,34}$ or incorporated into polymer matrix. ${ }^{30}$ Further, it is found that the incorporation of GO into polymer matrix membranes can improve obviously water permeation, ${ }^{35}$ antifouling $^{36}$ and salt rejection ${ }^{37}$ in desalination. Therefore, considering the hydrophilic nature of GO and the mechanical strength of polymer, the incorporation of GO into polymer to fabricate GO-based mixed matrix membranes (MMMs) is promising strategy to improve the mechanical performance of GO membranes.

Polyimide (PI), with excellent heat resistance, chemical compatibility and resistance over wide range of $\mathrm{pH}$, are widely used in the high temperature fuel cells, displays, separation 


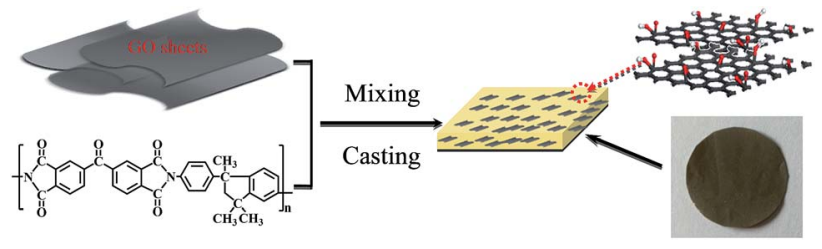

PI

GO/PI MMMs

Fig. 1 Schematic diagram of the preparation of GO/PI mixed matrix membranes (MMMs) through a phase inversion method.

membrane. ${ }^{38}$ In the present work, GO/PI MMMs is developed for seawater desalination by the incorporation of GO into PI matrix polymer through a phase inversion method (Fig. 1). Due to the combination of both advantages of GO and PI, it can be expected that the GO/PI MMMs will display excellent water permeability and salt rejection for seawater desalination.

\section{Experimental}

\section{Materials}

All reagents were used as received without further purification: concentrated sulfuric acid $\left(\mathrm{H}_{2} \mathrm{SO}_{4}, \mathrm{AR}, 98 \%\right)$, sodium nitrate $\left(\mathrm{NaNO}_{3}, \mathrm{AR}, 99 \%\right)$, potassium permanganate $\left(\mathrm{KMnO}_{4}, \mathrm{AR}\right.$, 99.5\%) were purchased from Sinopharm Chemical Reagent Co. Ltd (Shanghai, China). Graphite powder (G; 2000 mesh), $\mathrm{N}$ methyl-2-pyrrolidinone (NMP, GC, 99.9\%) and hydrogen peroxide $\left(\mathrm{H}_{2} \mathrm{O}_{2}\right.$, AR, $\left.30 \mathrm{wt} \%\right)$ were supplied by Aladdin Co., Ltd. Polyimide (PI) resin powder was obtained from Alfa Aesar and was dried under vacuum before use (overnight at $100{ }^{\circ} \mathrm{C}$ ).

\section{Preparation of GO nanosheets and GO/PI MMMs}

GO aqueous suspensions was prepared by the modified Hummers' method ${ }^{39}$ by using flake graphite powder as starting material according to the procedure as reported in the literature..$^{33,40}$ The resulting GO aqueous dispersion was sonicated for $30 \mathrm{~min}$ followed by centrifugation at $8000 \mathrm{rpm}$ for $30 \mathrm{~min}$ to remove the unoxidized graphite. And then, the GO powder was obtained by freeze drying overnight.

Asymmetric GO/PI MMMs were prepared via phase inversion in a water coagulation bath. In brief, an amount of GO nanosheets $(0,0.5,1,2 \mathrm{wt} \%$, based on the weight of NMP and the casting solution) were dispersed in $5 \mathrm{ml} \mathrm{NMP}$ by ultrasonication for $30 \mathrm{~min}$. After 10 minutes of drastic stirring, $1.564 \mathrm{~g}$ PI was added into the GO solution sequentially at $50{ }^{\circ} \mathrm{C}$ with vigorous stirring. The obtained homogenous casting solution was allowed to settle overnight to remove air bubbles. After the solution was fully degassed, the GO/PI solution was cast on a glass plate by a casting knife with a $100 \mu \mathrm{m}$ thickness. Subsequently, the glass plate was immediately immersed into the water bath at ambient temperature. After primary phase separation and membrane formation, the GO/PI MMMs was peeled off carefully from the glass plate and immersed into fresh distilled water for $24 \mathrm{~h}$ to ensure complete phase separation. The hybrid membrane was dried at $60^{\circ} \mathrm{C}$ for $1 \mathrm{~h}$, and then stored for next characterizations and seawater desalination. Pristine PI membranes were also synthesized via similar procedure without addition of GO nanosheets. The resultant membranes were denoted as PI, 0.5 wt\%-GO/PI, 1 wt\%-GO/PI and $2 \mathrm{wt} \%-\mathrm{GO} / \mathrm{PI}$, respectively.

\section{Characterization of GO and GO/PI MMMs}

Fourier transform infrared spectroscopy (FT-IR, Bruker Tensor 27) was used to characterize the surface properties of the GO, PI and GO/PI MMMs. X-ray diffraction (XRD) was used to characterize the microstructures of GO, PI and GO/PI MMMs. XRD analyses were recorded on a Bruker D8 Advance X-ray diffractometer with $\mathrm{Cu} \mathrm{K} \alpha$ radiation at $40 \mathrm{kV}$ and $40 \mathrm{~mA}$. The X-ray photoelectron spectroscopy (XPS) was employed to characterize the elementary compositions of the GO sheets. Scanning electron microscopy (SEM, Carl Zeiss, EVO 18) was used to examine the morphology and thickness of the GO/PI MMMs. The static water contact angles of hybrid membranes were determined using an OCA20 contact angles system (Data Physics Co., Germany). The mechanical properties of the GO/PI MMMs were evaluated by measuring tensile strength and Young's modulus by an Instron 5567 machine at a constant speed of $5 \mathrm{~mm} \mathrm{~min}^{-1}$.

\section{Evaluation of desalination performances of the GO/PI MMMs}

The separation performances of the GO/PI MMMs for desalination of different concentrations of seawater were measured at different temperatures by pervaporation. ${ }^{27,41}$ The GO/PI MMMs with an effective area $1.13 \mathrm{~cm}^{2}$ were sealed into a membrane model by O-ring with the membrane surface facing the feed solution. The seawater, prepared by dissolving a specified amount of sea salts into distilled water with concentrations of 2-10 wt\%, was fed to the membrane side. In order to evaluate the effect of operation temperature on the separation performances of the GO/PI MMMs, the seawater was preheated to 45$90{ }^{\circ} \mathrm{C}$. The permeate side of the membrane was evacuated to $0.1 \mathrm{MPa}$ with a vacuum pump. Two freezing traps with liquid $\mathrm{N}_{2}$ cooling were used to collect the permeate at every time interval. For the determination of ion rejection, the permeate side of the membrane was washed periodically after certain times with deionized water to collect possibly permeated salts which have been deposited as crystals on the top or inside of the porous alumina supports. ${ }^{42}$ In the entire study, no salt precipitation or crystallization was observed on the permeate side of the GO/PI MMMs.

The performance of seawater desalination was assessed in terms of permeate flux $(F)$ and total ion rejection $\left(R_{\mathrm{i}}\right)$. The water flux, $F\left(\mathrm{~kg} \mathrm{~m}^{-2} \mathrm{~h}^{-1}\right)$, was calculated by eqn (1):

$$
F=\frac{W}{\Delta t \cdot A}
$$

where $W$ is the permeate mass (kg), $\Delta t$ is collecting time (h), $A$ is the membrane area $\left(\mathrm{m}^{2}\right)$.

The total ion rejection was defined as the ratio of the ion conductivity in the permeate to that in the feed, and given by eqn (2): 


$$
R_{\mathrm{i}}=\frac{S_{\mathrm{if}}-S_{\mathrm{ip}}}{S_{\mathrm{if}}} \times 100 \%
$$

where $S_{\text {if }}$ and $S_{\text {ip }}$ are the ion conductivities in the feed and permeate, respectively. Both $S_{\text {if }}$ and $S_{\text {ip }}$ were analyzed after water dilution by 100 times. The ions conductivities of the feed and the permeate were measured by conductivity meter (DDSJ308A, Shanghai REX Instrument Factory).

The ion rejection for every ion was defined as the ratio of the ion concentration in the permeate to that in the feed, and given by eqn (3):

$$
R_{\mathrm{i}}=\frac{C_{\text {if }}-C_{\text {ip }}}{C_{\text {if }}} \times 100 \%
$$

where $C_{\text {if }}$ and $C_{\text {ip }}$ are the ion concentration in the feed and permeate, respectively. The concentrations of cations $\left(\mathrm{Na}^{+}\right.$, $\left.\mathrm{NH}_{4}{ }^{+}, \mathrm{K}^{+}, \mathrm{Ca}^{2+}, \mathrm{Mg}^{2+}\right)$ and anions $\left(\mathrm{Cl}^{-}, \mathrm{NO}_{3}{ }^{-}\right.$and $\left.\mathrm{SO}_{4}{ }^{2-}\right)$ in the feed and the permeate were analyzed by ion chromatography (Dionex Corporation, USA) with an AS-19 column and ASRS 4 mm suppressor.

\section{Results and discussion}

\section{Preparation and characterization of GO nanosheets and GO/PI MMMs}

Fig. 2a shows the FT-IR spectra of GO, which indicates the presence of hydroxyl groups (-OH at $\left.3388 \mathrm{~cm}^{-1}\right)$, carboxyl groups $\left(\mathrm{C}=\mathrm{O}\right.$ at $1726 \mathrm{~cm}^{-1}$ and $\mathrm{C}-\mathrm{OH}$ at $\left.1382 \mathrm{~cm}^{-1}\right)$, and unoxidized aromatic $\mathrm{C}=\mathrm{C}$ at $1627 \mathrm{~cm}^{-1}$, epoxy groups $(\mathrm{C}-\mathrm{O}-\mathrm{C}$ at $1242 \mathrm{~cm}^{-1}$ ) and alkoxy groups (C-O at $1066 \mathrm{~cm}^{-1}$ ) resulting from the oxidation process. ${ }^{43-45}$ The variety of functional groups such as epoxy, hydroxyl groups on the surface and carbonyl groups on the edge of GO sheets lead to the hydrophilic nature of GO.

Fig. 2b shows the FT-IR spectra of PI. The obvious absorption peaks at about $1779 \mathrm{~cm}^{-1}$ and $1725 \mathrm{~cm}^{-1}$ are attribute to the asymmetric and symmetric stretching of $\mathrm{C}=\mathrm{O}$ groups in imide groups. The band at $1383 \mathrm{~cm}^{-1}$ corresponds to the $\mathrm{C}-\mathrm{N}$

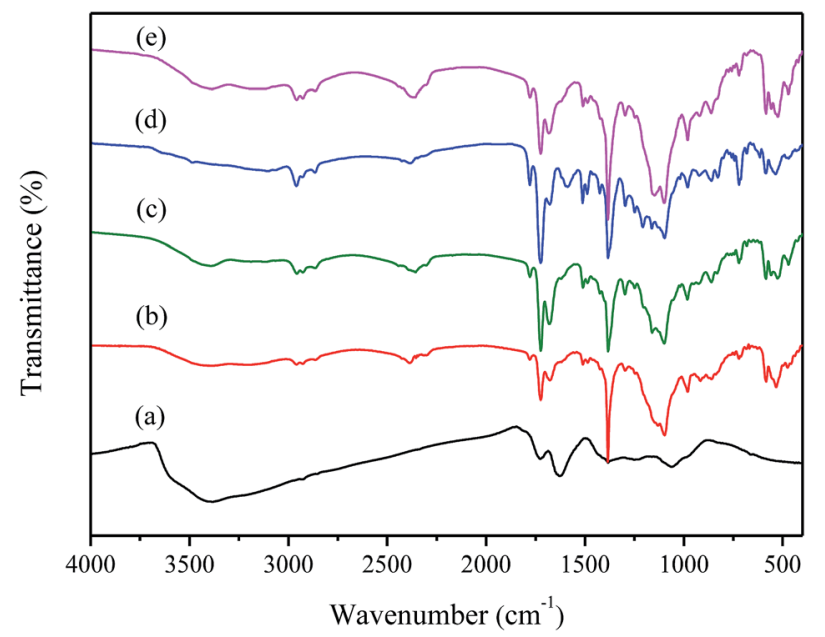

Fig. 2 FT-IR spectra of GO (a), PI (b), 0.5 wt\% GO/PI (c), 1 wt\% GO/PI (d), 2 wt\% GO/PI (e) MMMs. stretching in the imide group. Apart from this, the weak band at $1679 \mathrm{~cm}^{-1}$ is the $\mathrm{C}=\mathrm{O}$ stretch of amide groups, and the band at $1510 \mathrm{~cm}^{-1}$ is $\mathrm{C}-\mathrm{N}$ stretching of the $\mathrm{C}-\mathrm{N}-\mathrm{H}$ group. ${ }^{46-48}$ From Fig. 2c-e, the spectra of $0.5 \mathrm{wt} \% \mathrm{GO} / \mathrm{PI}, 1 \mathrm{wt} \% \mathrm{GO} / \mathrm{PI}$ and $2 \mathrm{wt} \%$ $\mathrm{GO} / \mathrm{PI} \mathrm{MMMs}$, the characteristic peaks of GO are increased with the increase of GO loading, confirming the dispersion of GO in polyimide matrix. The peaks of $\mathrm{GO}$ at $1382 \mathrm{~cm}^{-1}$ of $\mathrm{O}-\mathrm{H}$ depressed greatly, accompanied by increasing intensity the $\mathrm{C}-\mathrm{O}-\mathrm{C}$ stretching vibration of GO at $1066 \mathrm{~cm}^{-1} \cdot{ }^{47}$ The change of intensities of adsorption confirms the reactions between GO and PI. The XPS spectra of GO is shown in Fig. S1, $\uparrow$ four peaks at binding energies of 284.8, 286.8, 288.2 and $289.4 \mathrm{eV}$ are clearly observed, which correspond with $\mathrm{C}-\mathrm{C} / \mathrm{C}=\mathrm{C}, \mathrm{C}-\mathrm{O} / \mathrm{C}-\mathrm{O}-\mathrm{C}, \mathrm{C}=\mathrm{O}$ and $\mathrm{O}-\mathrm{C}=\mathrm{O}$, respectively. ${ }^{45,49}$ And an $\mathrm{O} / \mathrm{C}$ ratio of around 0.54 is found for the GO sheets, which is consistent with the previous reports. ${ }^{34,50}$ The results from XPS and FT-IR spectra agree well with the Lerf-Klinowski Model of GO. ${ }^{51}$

The XRD spectra of GO, pure PI as well as GO/PI MMMs was shown in Fig. 3. As shown in Fig. 3a, the feature diffraction peak at $2 \theta=10.76^{\circ}$ corresponds to GO powder. The calculated average interlayer spacing of GO is $0.82 \mathrm{~nm}$. The variation of peak position and $d$-spacing can be attributed to the intercalating oxygenated functional groups, which increased the disorder of structural after oxidation reaction. In addition, the absorption of water also enlarging the interlayer spacing of GO. ${ }^{29,52}$ From Fig. 3 b, it can be seen that the XRD pattern of PI exhibits a broad peak at around $15.6^{\circ}$ due to its amorphous nature. When GO was incorporated into the PI matrix, although the XRD patterns of GO/PI composites are almost as same as that of pure PI, a weak peak presented at $10^{\circ}$ in the $\mathrm{GO} / \mathrm{PI}$ MMMs is observed, suggesting the GO is dispersed in the entire membrane (Fig. 3c-e).

Fig. 4 shows the SEM images of the GO/PI MMMs with different GO content. As shown in Fig. 4a, there are many macro

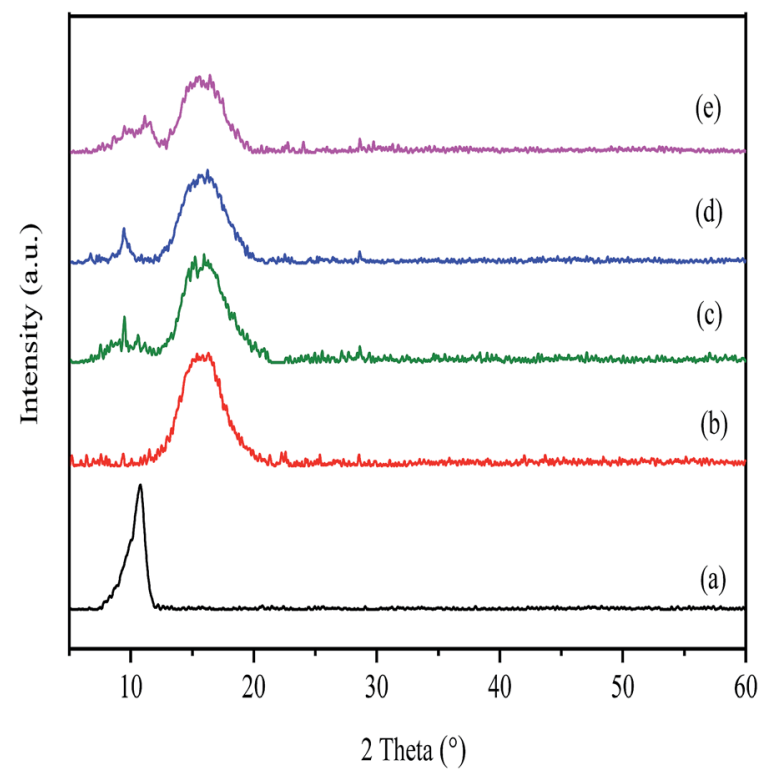

Fig. 3 XRD patterns of the GO (a); PI (b); and GO/PI MMMs with 0.5 $w t \%$ (c); 1 wt\% (d), 2 wt\% (e) GO loading. 

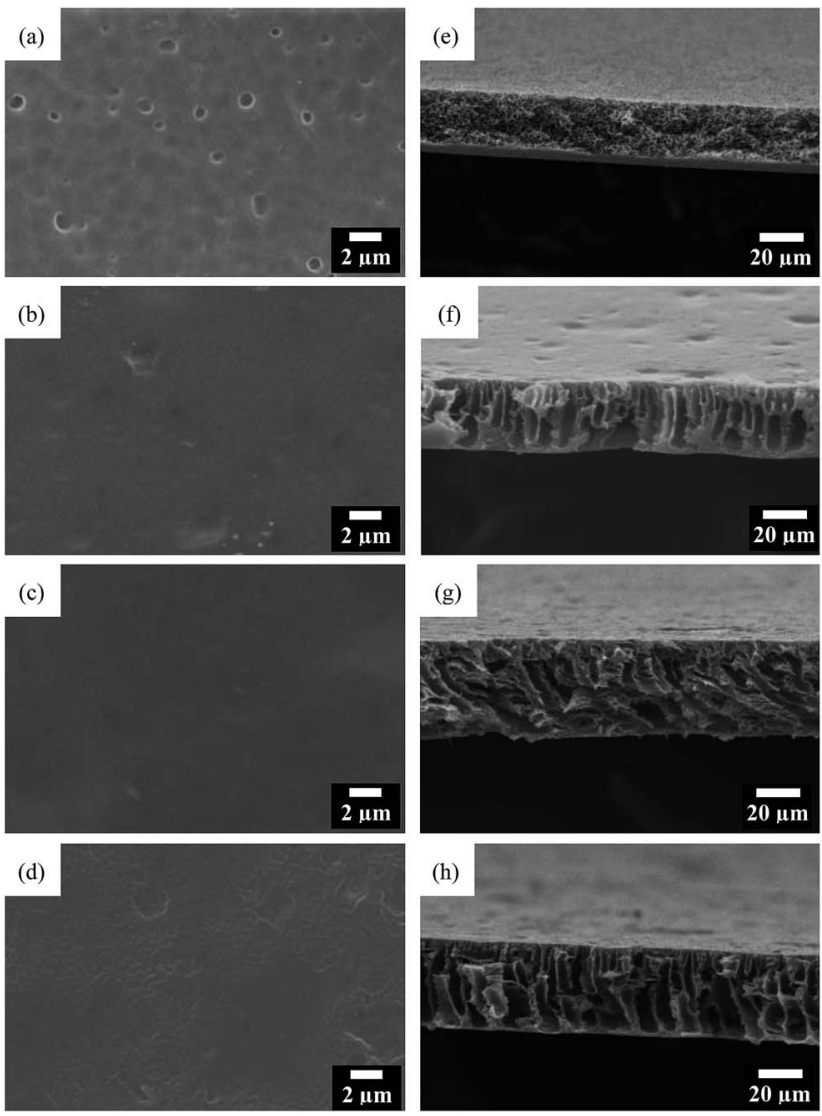

Fig. 4 Top view $(a-d)$ and cross-section (e-h) SEM images of the PI membrane (a and e) and GO/PI MMMs with 0.5 wt\% (b and f); 1 wt\% (c and g), 2 wt\% (d and h) GO loading.

pores on the surface of the pure PI membrane. After the introduction of GO nanosheets, the surfaces of the membranes are smooth, and no cracks, pinholes or other macroscopic defects can be observed (Fig. $4 \mathrm{~b}$ and c). Further, it is found that GO are homogeneously dispersed in the PI matrix without aggregation in the MMMs. However, some micropores are observed when the GO content is increased to $2 \mathrm{wt} \%$ (Fig. 4d), which confirms that high GO concentration leads to high dope viscosity, thereby slowing down the movement of GO sheets to the membrane surfaces. As reported previously, ${ }^{53} \mathrm{GO}$ with high surface polarity shows better dispersion in the PI polymer matrix, but the aggregation will occur with the added GO content more than $3 \mathrm{wt} \%$.

The cross-section SEM images of GO/PI MMMs are shown in Fig. $5 f-h$. It can be seen that there are no obvious differences for all GO/PI MMMs. Typically, an asymmetric porous structure is formed containing a dense skin top-layer and a fingerlike porous sub-layer for all GO/PI MMMs. But the pure PI membrane only has a sponge-like porous structure. These results indicate that the GO incorporation plays an important role in the formation of a dense asymmetric membrane since the hydrophilic groups on GO can accelerate the rate of solvent and non-solvent exchange during phase-inversion process. ${ }^{35}$ Consequently, finger-like porous sub-layer is formed instead of the formation of sponge structure. It is interesting to find that

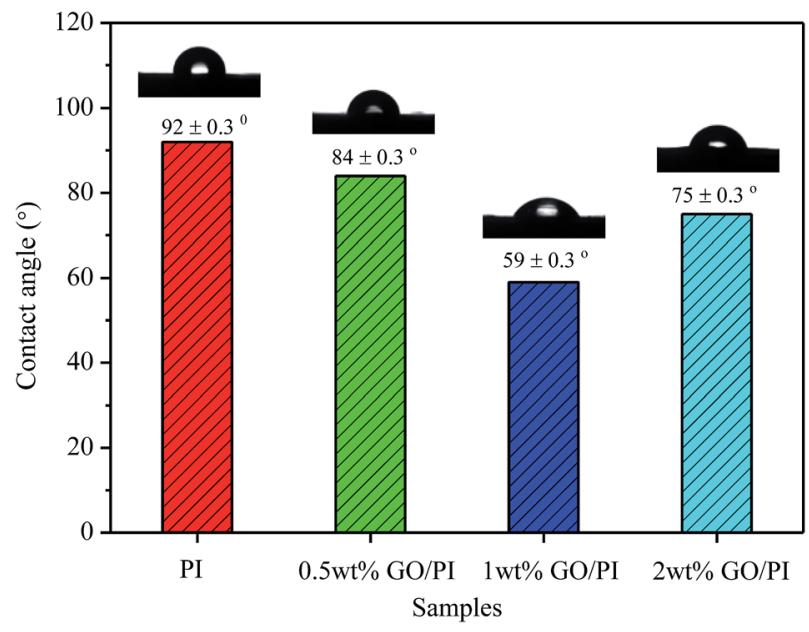

Fig. 5 Water contact angle (CA) of PI membrane and GO/PI MMMs prepared with different $\mathrm{GO}$ loading.

lateral pore structures are observed within the GO/PI MMMs when the GO content increases up to $1 \mathrm{wt} \%$, which is helpful to enhance the water fluxes of the GO/PI MMMs due to the remarkable reduction of the resistance of mass transfer. Judging by the cross-section morphologies, the thickness of the $d \mathrm{GO} / \mathrm{PI}$ MMMs is approximately $46 \mu \mathrm{m}$, about one or two orders of magnitude thicker than the traditional pure GO membranes, ${ }^{18,43}$ which is beneficial to enhance the mechanical strength of the GO membrane.

The water contact angle (CA) on the membranes surface was employed to measure the hydrophilicity of the GO/PI MMMs. As shown in Fig. 5, the water CA of the pure PI membrane is about $92^{\circ}$. When the GO is incorporated into the PI matrix, water CA of the GO/PI MMMs decreases due to the introduction of hydrophilic groups. As the GO loading increases to $1 \mathrm{wt} \%$ in the PI matrix, the water CA of the GO/PI MMMs decreases to $59^{\circ}$, indicating a more hydrophilic membrane surface. This phenomenon results from the migration of GO nanosheets towards the top surface of the membrane during the phase inversion process. ${ }^{37}$ However, the water CA of the GO/PI MMMs increases to $75^{\circ}$ when the GO content surpasses to $2 \mathrm{wt} \%$. Probably, with GO content up to $2 \mathrm{wt} \%$, the viscosity of the casting solution is too high to reduce the exchange of solvent and non-solvent during the phase-inversion process. Consequently, the GO nanosheets cannot move to the surface of the membrane immediately, resulting in an increase of contact angle. ${ }^{35}$

The mechanical properties of the GO/PI MMMs were evaluated by tensile strength and elongation tests, which are shown in Fig. S2. $\dagger$ As shown in Fig. S2, $\dagger$ the tensile strengths of all of the composite membranes are more than $9.3 \mathrm{MPa}$, which is much higher than previously reported GO blended polymer membranes and GO membranes, ${ }^{30,35,54}$ suggesting that when the GO is introduced into the polymer, the formation of the finger-like pores results in slight reduction of the tensile strengths of the GO/PI MMMs. Comparing with the pure PI membrane, the Young's modulus of the GO/PI MMMs is 
obviously enhanced from $311 \mathrm{MPa}$ to $382 \mathrm{MPa}$. The Young's modulus of the GO/PI MMMs increases with the increase of GO content due to the very high aspect ratio of the graphene oxide. ${ }^{36}$ These results demonstrate that the elasticity of the GO/PI MMMs will decline with the adding of GO into the PI matrix. The good compatibility between GO and PI results in a homogeneous dispersion of GO within the PI matrix, which restricts the polymer chain mobility and thus leading to a concomitant improvement in the membrane Young's modulus. ${ }^{55,56}$

\section{Evaluation of the desalination performance of the GO/PI MMMs}

The desalination performances of the $1 \mathrm{wt} \%$ GO/PI MMMs were evaluated by pervaporation. Fig. 6 shows the water flux and ion rejection of the GO/PI MMMs as a function of the operation temperature for the desalination of $3.5 \mathrm{wt} \%$ seawater. It can be seen that, in the temperature range of $45-90{ }^{\circ} \mathrm{C}$, all the ion rejections are over 99.9\%, indicating that the GO/PI MMMs display excellent desalination performances. In fact, the ion rejection of $\mathrm{Na}^{+}, \mathrm{NH}_{4}{ }^{+}, \mathrm{K}^{+}, \mathrm{Mg}^{2+}, \mathrm{Ca}^{2+}, \mathrm{Cl}^{-}, \mathrm{NO}_{3}{ }^{-}, \mathrm{SO}_{4}{ }^{2-}$ is 99.99, 100.00, 99.98, 99.97, 99.95, 99.95, 99.91, 99.86\%, respectively (Table S1 $\uparrow$ ). The excellent desalination performance of the GO/PI MMMs is attributed to the introduction of plenty of hydrophilic sites, which facilitate the sorption and diffusion of water molecules. On the contrary, when PI membrane is not incorporated with GO, the PI membranes display much lower flux than that of GO/PI MMMs for desalination of $3.5 \mathrm{wt} \%$ seawater (Fig. S3†). It is found that the water flux of the $0.5 \mathrm{wt} \%$ or $2 \mathrm{wt} \% \mathrm{GO} / \mathrm{PI}$ MMMs are much lower than those of the $1 \mathrm{wt} \%$ GO/PI MMMs although all GO/PI MMMs show a high ion rejection (Fig. S4†).

To evaluate the thermal stability of the GO/PI MMMs, the GO/ PI MMMs was subjected to seawater desalination at different temperatures and for a long operation time. As shown in Fig. 6, with an increase in the feed temperature from 45 to $90{ }^{\circ} \mathrm{C}$, the water flux increases from $14.9 \mathrm{~kg} \mathrm{~m}^{-2} \mathrm{~h}^{-1}$ to $36.1 \mathrm{~kg} \mathrm{~m}^{-2} \mathrm{~h}^{-1}$, and the salt rejections are maintained unchanged (99.9\%). This result is in good agreement with the thermodynamics theory.

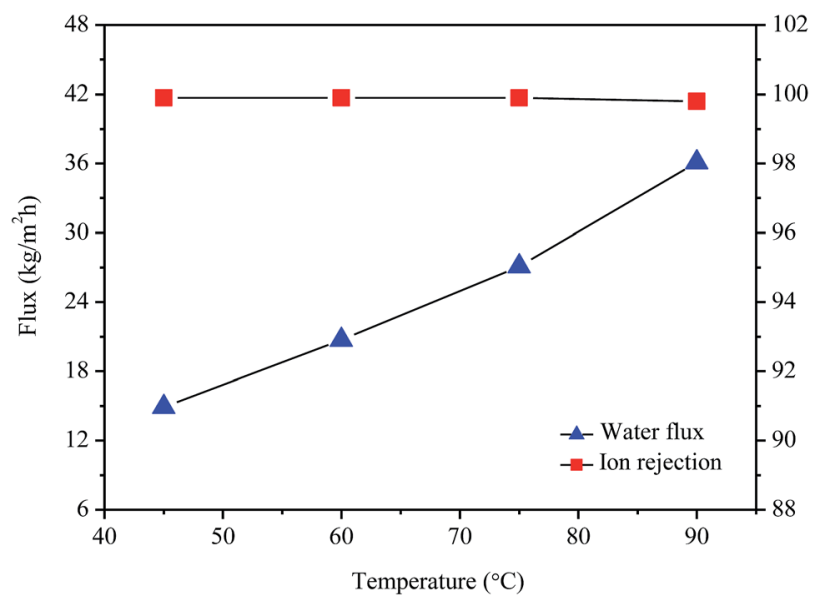

Fig. 6 Water flux and ion rejection of the GO/PI MMMs with 1 wt\% GO loading as a function of the operation temperature for desalination of $3.5 \mathrm{wt} \%$ seawater by pervaporation.
When the feed temperature increases, the water vapour pressure on the feed side increases exponentially, while the vapour pressure on the permeate side keeps unchanged, thus resulting in an enhancement of the driving force across the membrane. On the other hand, the thermal motion of water molecules is agitated with the increase of temperature, thus the diffusion rate of water molecules through the GO/PI MMMs is also accelerated. Therefore, the water flux of the GO/PI MMMs increases with increasing of temperature. In fact, from an Arrhenius type plot (eqn (4)) with $\ln$ (flux) versus $1 / \mathrm{T}$ (Fig. $\mathrm{S} 5 \dagger$ ), the water permeation through the GO/PI MMMs is a typically activated diffusion process. Based on the Arrhenius equation, the apparent activation energy $\left(E_{\text {act }}\right)$ of water permeation in the GO/PI MMMs is about 18.7 $\mathrm{kJ} \mathrm{mol}{ }^{-1}$, which is lower than that of the GO membranes. ${ }^{26,27}$ Thus, the water molecules can easily pass through the GO/PI MMMs.

$$
\ln J=9.77-\frac{18.7 \times 10^{3}}{R T}
$$

Fig. 7 shows the effect of the feed concentration on the water flux and ion rejection of the GO/PI MMMs membrane. As shown in Fig. 7 , at $75^{\circ} \mathrm{C}$, when the feed concentration increases from 2 to $10 \mathrm{wt} \%$, the water flux decreases from 22.4 to $10.7 \mathrm{~kg} \mathrm{~m}^{-2}$ $\mathrm{h}^{-1}$, while the ion rejection rate still maintains high (over $99.8 \%$ ) even for desalination of $10 \mathrm{wt} \%$ seawater. This result is consistent with previous reports. ${ }^{\mathbf{1 1 , 4 0 , 5 7}}$ The process of salt ions passing through the pores is usually determined by the pore size and electrostatic interactions between ions and membrane pores. When the feed concentration increases, the concentration polarization will take place at the interface between the feed solution and the membrane. ${ }^{42}$ Therefore, more salts are deposited on the outer surface of the membranes with the increase in salt concentration, and thus the electrostatic interactions increases, resulting in a great reduction of water activity and a significant drop in water flux. ${ }^{58}$ In fact, a higher water flux can be observed through the $1 \mathrm{wt} \%$ GO/PI MMMs for permeation of pure water (Fig. S6†).

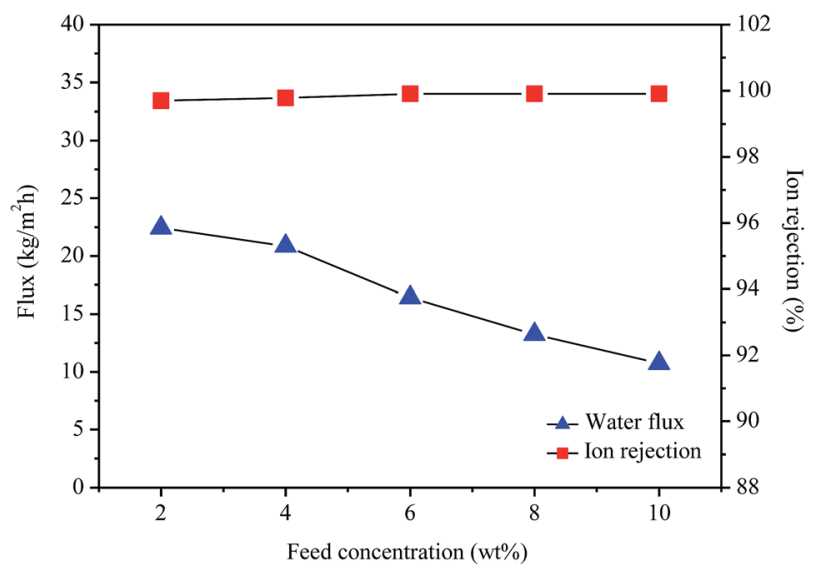

Fig. 7 Water flux and ion rejection of the GO/PI MMMs as a function of the feed concentration for desalination of different concentration seawater by pervaporation. 


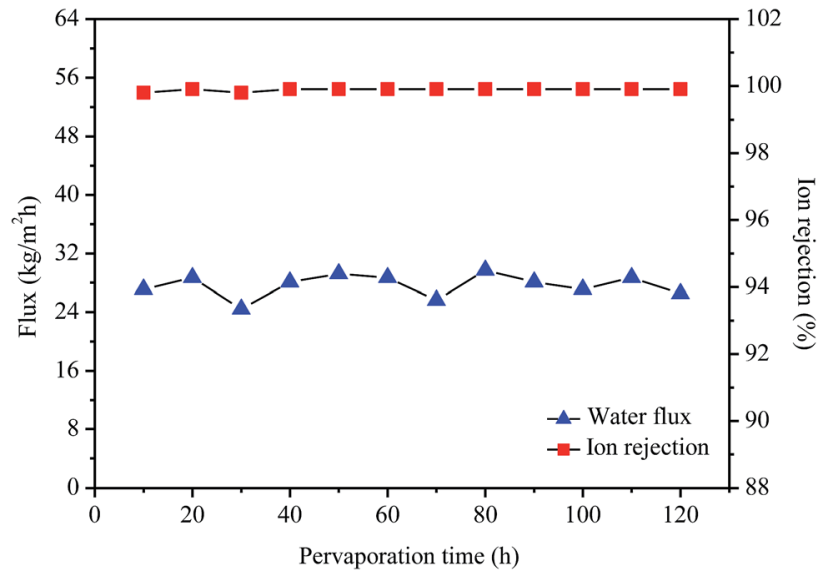

Fig. 8 Stability measurement of the GO/PI MMMs for desalination of $3.5 \mathrm{wt} \%$ seawater by pervaporation at $75^{\circ} \mathrm{C}$.

Fig. 8 shows stability measurement of the GO/PI MMMs for desalination of $3.5 \mathrm{wt} \%$ seawater by pervaporation at $75{ }^{\circ} \mathrm{C}$. As shown in Fig. 8, high desalination performances of the GO/PI MMMs can keep unchanged for a long time, both the ion rejection and water flux are almost constant after $120 \mathrm{~h}$ at $75^{\circ} \mathrm{C}$, indicating that the GO/PI MMMs displays high stability for seawater desalination due to the formation of strong covalent bonds among the oxygen-containing functional groups on GO nanosheets and polyimide resin matrix. Further, since the preparation of the GO/PI MMMs is easily controlled, the reproducibility of the preparation of GO/PI MMMs is high, and about 90\% GO/PI MMMs display similar separation performances.

\section{Conclusions}

In conclusion, thin GO/PI MMMs have been prepared by a wet phase inversion method. The GO nanosheets were homogeneously dispersed in the PI hybrid membrane and without aggregation in the mixed matrix membrane. Under the same thickness, the tensile strength of the GO/PI MMMs was significantly enhanced. The hydrophilic properties of GO/PI MMMs improved significantly after the introduction of GO because a plenty of oxygen-containing groups were distributed on the surface of GO. The GO/PI MMMs showed high water permeability and ion rejection even for desalination of $10 \mathrm{wt} \%$ seawater. At $90{ }^{\circ} \mathrm{C}$, the GO/PI MMMs exhibit a high permeation flux $\left(36.1 \mathrm{~kg} \mathrm{~m}^{-2} \mathrm{~h}^{-1}\right.$ ) and a high ion rejection (about 99.9\%). The desalination performances of GO/PI MMMs kept unchanged for $120 \mathrm{~h}$ at $75{ }^{\circ} \mathrm{C}$. These excellent properties recommend the robust GO/PI MMMs as a promising candidate for desalination of seawater and brackish water.

\section{Acknowledgements}

Financial support by National Natural Science Foundation of China (21576273, 51402320), External Cooperation Program of BIC, CAS (174433KYSB2013005), and Ningbo Science and Technology Innovation Team (2014B81004).

\section{References}

1 N. R. Singh, J. Membr. Sci., 2008, 313, 353-354.

2 J. Zhu, M. Tian, J. Hou, J. Wang, J. Lin, Y. Zhang, J. Liu and B. Van der Bruggen, J. Mater. Chem. A, 2016, 4, 1980-1990.

3 X. Sun, B. Guo, L. He, P. Xia and S. Wang, AIChE J., 2016, 62, 2154-2162.

4 S. K. Srivastava, M. Guix and O. G. Schmidt, Nano Lett., 2016, 16, 817-821.

5 J. Glater, S.-K. Hong and M. Elimelech, Desalination, 1994, 95, 325-345.

6 H. El-Saied, A. H. Basta, B. N. Barsoum and M. M. Elberry, Desalination, 2003, 159, 171-181.

7 B. Corry, J. Phys. Chem. B, 2008, 112, 1427-1434.

8 R. Das, M. E. Ali, S. B. A. Hamid, S. Ramakrishna and Z. Z. Chowdhury, Desalination, 2014, 336, 97-109.

9 B. Zhu, Z. Hong, N. Milne, C. M. Doherty, L. Zou, Y. S. Lin, A. J. Hill, X. Gu and M. Duke, J. Membr. Sci., 2014, 453, 126-135.

10 W. An, X. Zhou, X. Liu, P. W. Chai, T. Kuznicki and S. M. Kuznicki, J. Membr. Sci., 2014, 470, 431-438.

11 P. Swenson, B. Tanchuk, A. Gupta, W. Z. An and S. M. Kuznicki, Desalination, 2012, 285, 68-72.

12 Y. Zhu, K. M. Gupta, Q. Liu, J. Jiang, J. Caro and A. Huang, Desalination, 2016, 385, 75-82.

13 T. Humplik, J. Lee, S. C. O'Hern, B. A. Fellman, M. A. Baig, S. F. Hassan, M. A. Atieh, F. Rahman, T. Laoui, R. Karnik and E. N. Wang, Nanotechnology, 2011, 22, 292001.

14 A. K. Mishra and S. Ramaprabhu, Desalination, 2011, 282, 39-45.

15 D. Li and H. Wang, J. Mater. Chem., 2010, 20, 4551-4566.

16 F. Perreault, A. Fonseca de Faria and M. Elimelech, Chem. Soc. Rev., 2015, 44, 5861-5896.

17 B. Mi, Science, 2014, 343, 740-742.

18 R. K. Joshi, P. Carbone, F. C. Wang, V. G. Kravets, Y. Su, I. V. Grigorieva, H. A. Wu, A. K. Geim and R. R. Nair, Science, 2014, 343, 752-754.

19 H. Li, Z. Song, X. Zhang, Y. Huang, S. Li, Y. Mao, H. J. Ploehn, Y. Bao and M. Yu, Science, 2015, 342, 95-98.

20 H. W. Kim, H. W. Yoon, B. M. Yoo, J. S. Park, K. L. Gleason, B. D. Freeman and H. B. Park, Chem. Commun., 2014, 50, 13563-13566.

21 H. W. Kim, H. W. Yoon, S.-M. Yoon, B. M. Yoo, B. K. Ahn, Y. H. Cho, H. J. Shin, H. Yang, U. Paik, S. Kwon, J.-Y. Choi and H. B. Park, Science, 2013, 342, 91-95.

22 Z. P. Smith and B. D. Freeman, Angew. Chem., Int. Ed., 2014, 53, 10286-10288.

23 J. Kim, L. J. Cote, F. Kim, W. Yuan, K. R. Shull and J. X. Huang, J. Am. Chem. Soc., 2010, 132, 8180-8186.

24 R. R. Nair, H. A. Wu, P. N. Jayaram, I. V. Grigorieva and A. K. Geim, Science, 2012, 335, 442-444.

25 W.-S. Hung, C.-H. Tsou, M. De Guzman, Q.-F. An, Y.-L. Liu, Y.-M. Zhang, C.-C. Hu, K.-R. Lee and J.-Y. Lai, Chem. Mater., 2014, 26, 2983-2990.

26 B. Feng, K. Xu and A. Huang, Desalination, 2016, 394, 123130. 
27 K. Xu, B. Feng, C. Zhou and A. Huang, Chem. Eng. Sci., 2016, 146, 159-165.

28 C. N. Yeh, K. Raidongia, J. Shao, Q. Yang and J. Huang, Nat. Chem., 2015, 7, 166-170.

29 N. F. D. Aba, J. Y. Chong, B. Wang, C. Mattevi and K. Li, J. Membr. Sci., 2015, 484, 87-94.

30 M. Ionita, A. M. Pandele, L. Crica and L. Pilan, Composites, Part B, 2014, 59, 133-139.

31 A. M. Pandele, S. Dinescu, M. Costache, E. Vasile, C. Obreja, H. Iovu and M. Ionita, Polym. Compos., 2013, 34, 2116-2124.

32 M. Ionita, M. A. Pandele and H. Iovu, Carbohydr. Polym., 2013, 94, 339-344.

33 C. A. Zuniga, J. B. Goods, J. R. Cox and T. M. Swager, ACS Appl. Mater. Interfaces, 2016, 8, 1780-1785.

34 J. Zhao, Y. Zhu, F. Pan, G. He, C. Fang, K. Cao, R. Xing and Z. Jiang, J. Membr. Sci., 2015, 487, 162-172.

35 Z. Wang, H. Yu, J. Xia, F. Zhang, F. Li, Y. Xia and Y. Li, Desalination, 2012, 299, 50-54.

36 J. Lee, H. R. Chae, Y. J. Won, K. Lee, C. H. Lee, H. H. Lee, I. C. Kim and J. M. Lee, J. Membr. Sci., 2013, 448, 223-230.

37 B. M. Ganesh, A. M. Isloor and A. F. Ismail, Desalination, 2013, 313, 199-207.

38 L. Diestel, N. Wang, B. Schwiedland, F. Steinbach, U. Giese and J. Caro, J. Membr. Sci., 2015, 429, 181-186.

39 W. S. Hummers Jr and R. E. Offeman, J. Am. Chem. Soc., 1958, 80, 1339.

40 K. S. Hu, M. K. Gupta, D. D. Kulkarni and V. V. Tsukruk, Adv. Mater., 2013, 25, 2301-2307.

41 K. Xu, Z. Jiang, B. Feng and A. Huang, RSC Adv., 2016, 6, 23354-23359.

42 M. C. Duke, J. O'Brien-Abraham, N. Milne, B. Zhu, J. Y. Lin and J. C. D. da Costa, Sep. Purif. Technol., 2009, 68, 343-350.

43 B. Liang, W. Zhan, G. G. Qi, S. S. Lin, Q. Nan, Y. X. Liu, B. Cao and K. Pan, J. Mater. Chem. A, 2015, 3, 5140-5147.
44 Y. P. Tang, D. R. Paul and T. S. Chung, J. Membr. Sci., 2014, 458, 199-208.

45 S. J. Xia, M. Ni, T. R. Zhu, Y. Zhao and N. N. Li, Desalination, 2015, 371, 78-87.

46 J. Hu, H. Cai, H. Ren, Y. Wei, Z. Xu, H. Liu and Y. Hu, Ind. Eng. Chem. Res., 2010, 49, 12605-12612.

47 J. Han, W. Lee, J. M. Choi, R. Patel and B. R. Min, J. Membr. Sci., 2010, 351, 141-148.

48 H. Liu, Y. Li, T. Wang and Q. Wang, J. Membr. Sci., 2011, 47, 1867-1874.

49 L. Huang, Y. Li, Q. Zhou, W. Yuan and G. Shi, Adv. Mater., 2015, 27, 3797-3802.

50 D. C. Marcano, D. V. Kosynkin, J. M. Berlin, A. Sinitskii, Z. Sun, A. Slesarev, L. B. Alemany, W. Lu and J. M. Tour, ACS Nano, 2010, 4, 4806-4814.

51 H. He, T. Riedl, A. Lerf and J. Klinowski, J. Phys. Chem., 1996, 100, 19954-19958.

52 J. T. Chen, Y. J. Fu, Q. F. An, S. C. Lo, S. H. Huang, W. S. Hung, C. C. Hu, K. R. Lee and J. Y. Lai, Nanoscale, 2013, 5, 9081-9088.

53 L. Zhao, C. Cheng, Y. F. Chen, T. Wang, C. H. Du and L. G. Wu, Polym. Adv. Technol., 2015, 26, 330-337.

54 L. Yang, B. B. Tang and P. Wu, J. Mater. Chem. A, 2014, 2, 18562-18573.

55 C. Zhao, X. Xu, J. Chen and F. Yang, Desalination, 2014, 334, 17-22.

56 M. El Achaby, F. Z. Arrakhiz, S. Vaudreuil, E. M. Essassi and A. Qaiss, Appl. Surf. Sci., 2012, 258, 7668-7677.

57 B. Liang, K. Pan, L. Li, E. P. Giannelis and B. Cao, Desalination, 2014, 347, 199-206.

58 J. Yang, H. Li, J. Xu, J. Wang, X. Meng, K. Bai, J. Lu, Y. Zhang and D. Yin, Microporous Mesoporous Mater., 2014, 192, 60-68. 